\title{
COMENTARIO PEDAGÓGICO PASTORAL DE LA CARITAS IN VERITATE DE BENEDICTO XVI
}

Juan Alberto Osorio Torres*

\begin{abstract}
RESUMEN
Caritas in veritate (2009), es la primera carta del Papa Benedicto XVI, dedicada específicamente a la Doctrina Social de la Iglesia, después de haber escrito otras dos, desde el comienzo de su pontificado: Deus caritas est (2006) y Spes Salvi (2007). Para elaborar este artículo, fue necesario revisar el texto Jesús de Nazareth (2007), el primer volumen publicado por el Papa Teólogo, a fin de captar la unidad y la coherencia de su pensamiento; pues, tanto en la segunda parte de la Deus caritas est, antes de enumerar las encíclicas sociales, desde la Rerum Novarum a la Centesimus Annus y de esta a la publicación del Compendio de la DSI, el Papa habla de la caridad como tarea de la iglesia, le dedica un apartado especial al tema de la justicia y la política, y hace importantes precisiones acerca de la relación entre justicia y caridad, temas para tener en cuenta en la pedagogía y la pastoral.
\end{abstract}

\section{PALABRAS CLAVE:}

Doctrina Social de la Iglesia, Justicia Social, Politica, Caridad, Reino de Dios, Vocación de la Iglesia

\section{PASTORAL ETEACHING COMMENTS ON "CARITAS IN VERITATE" BY BENEDICTO XVI}

\begin{abstract}
Caritas in Veritate (2009) is the first encyclical letter of Pope Benedict XVI, dedicated specifically to the Social Doctrine of the Church, after having written two others, from the beginning of his pope, Deus Caritas Est (2006) and Spes Salvi(2007). To prepare this article, it was necessary to revise the text Jesus of Nazareth (2007), the first volume published by the Theologian Pope, so to capture the unity and coherence of his thought. Because, in the second part of Deu scaritas est, before listing the social encyclical letters from Rerum Novarum to Centesimus Annus and from this to the publication of Compendium of the Church's Social Doctrine, the Pope speaks of charity as the responsibility of the church, he dedicates a special section to justice and politics, and makes important remarks about the relations hip between justice and charity, issues that should be considered in teaching and pastoral care.
\end{abstract}

\section{KEYWORDS:}

Social Doctrine of the Church, Social Justice, Politics, Charity, Kingdom of God, the Church's Vocation

\footnotetext{
* Sacerdote de la Diócesis de Cajamarca, prestando sus servicios a la Diócesis de Lurín en Lima. Magíster en Antropología y candidato a Doctor en esta especialidad en la Pontificia Universidad Católica del Perú. Miembro del Seminario Interdisciplinar de Estudios Religiosos (SIER) de la PUCP. Profesor de Teología y Capellán de la UNIFÉ. Actual Vicario de la Parroquia Sagrado Corazón de Pamplona Alta, responsable parroquial de los Asentamientos Humanos de la Nueva Rinconada de Pamplona.
} 
$\square$ n un acápite de la Deus caritas est, Benedicto XVI comparte una percepción con clara motivación pastoral, y por eso discretamente autocrítica. Allí, desde el rol universal que estrenó a partir de sus 78 años, y ya en un complicado y agobiado comienzo del siglo XXI, deja brotar en el estilo y contenido que le vamos conociendo, la inquietud del Pastor, que no encubre ni silencia la demora o el insuficiente cuidado pastoral en el comportamiento jerárquico de la Iglesia del siglo XIX: "Se debe admitir -advierte- que los representantes de la Iglesia percibieron sólo lentamente que el problema de la estructura justa de la sociedad se planteaba de un modo nuevo."(DCE.27)

El reconocimiento papal de ese grave tardío modo de percibir el rumbo y el funcionamiento de la sociedad por parte de los líderes de una institución consagrada en exclusiva al servicio pastoral del mundo, puede ser una significativa muestra de la sincera lamentación del máximo líder de la Iglesia, que deplora la baja o insuficiente calidad de la caridad de los ministros de la Iglesia. Es tal vez, esta constatación del pasado, la que explica el empeño del Papa, por plantear y esclarecer para nuestro presente, a lo largo de todos sus recientes escritos sobre la Caridad, y poner en guardia por todos los medios el problema de "la verdad de una caridad ajena a la verdad y/o de una caridad desierta o vacía de verdad".

Comentamos a continuación dos aspectos que me parecen útiles para el tema pastoral además de centrales en el pensamiento del Papa, porque le permiten ayudarnos a conseguir una percepción auténtica de los actuales retos del cristianismo. El primero, referido a la caridad y el segundo, al empleo del término vocación.

\section{La caridad en la Caritas in Veritate del Papa Benedicto XVI}

Como no hay mejor manera de abordar la pastoral que preguntarnos por quien real y efectivamente encarnó el papel de pastor hasta el final y hasta sus consecuencias, inicio mi comentario, partiendo de algunos aspectos sobre la manera en que es planteada la presencia de Jesús, el Buen Pastor en la Caritas in veritate. $Y$ señalo a continuación las implicancias pastorales que se pueden desprender de tal enfoque. Para esto, constaté que, mientras en la Deus caritas est, la palabra 'pastor' solo es empleada dos veces, en la Spes Salvi, siete, y en Jesús de Nazareth, 78, sorprendentemente en la Caritas in veritate, tal expresión, juntamente con otras como 'reino de Dios', no figuran explícitamente en ningún momento. Por eso, dados los límites de esta presentación, doy cuenta global de la dimensión pastoral presente en esta su tercera encíclica, habiendo tenido que acudir a la Deus caritas est, en donde el Papa explica lo que él entiende por pastor. Más que una simple función, es una apuesta y una inversión vital. Cito: "Cuando Jesús habla en sus parábolas del pastor que va tras la oveja descarriada, de la mujer que busca el dracma, del padre que sale al encuentro del hijo pródigo y lo abraza, no se trata sólo de meras palabras, sino que es la explicación de su propio ser y actuar. En su muerte en la cruz se realiza ese ponerse Dios contra sí mismo, al entregarse para dar nueva vida al hombre y salvarlo: esto es amor en su forma más radical. Poner la mirada en el costado traspasado de Cristo, del que habla Juan (cf. 19, 37), ayuda a comprender lo que ha sido el punto de partida de esta Carta encíclica: 'Dios es amor' (1 Jn 4, 8). Es allí, en la cruz, donde puede contemplarse esta verdad. Y a partir de allí se debe definir ahora qué es el amor. Y, desde esa mirada, el cristiano 
encuentra la orientación de su vivir y de su amar." (DCE 12)

A la pregunta sobre quées el amor, quées la caridad, el Papa responde en la Caritas in Veritate de la manera siguiente: "La caridad es la vía maestra de la doctrina social de la Iglesia. Todas las responsabilidades y compromisos trazados por esta doctrina provienen de la caridad que, según la enseñanza de Jesús, es la síntesis de toda la Ley (cf. Mt 22,36-40). Ella da verdadera sustancia a la relación personal con Dios y con el prójimo; no es sólo el principio de las micro-relaciones, como en las amistades, la familia, el pequeño grupo, sino también de las macro-relaciones, como las relaciones sociales, económicas y políticas." (CV. 2)

Hoy, que un alto porcentaje de fieles, no sólo no frecuentan más las parroquias ni los templos, sino que no deslumbran por su sentido de pertenencia y mucho menos por testimonio público alguno de sus convicciones, el Papa vislumbra que para salir al encuentro de estos hermanos supuestamente "alejados", no puede bastar el impulso de la caridad si ella no es experimentada en el marco de la verdad. Un pastor, cuya caridad, no se encuentre autentificada o validada por la verdad que crea diálogos, comunicación y comunión, no estará comprometiendo a sus diócesis y sus parroquias en la inexcusable misión del creyente y ciudadano, de participar en la tarea de construir una buena sociedad y un verdadero desarrollo humano integral. Lejos de contribuir en la tarea de transformar la sociedad, la caridad reducida a simple emotividad y a peligroso fideísmo, no sólo conducirá a huir del compromiso cristiano sino que hará de esa caridad una legitimación del estado crítico en que se encuentra la actual sociedad, y una amenazadora sacralización de su actual funcionamiento.
En el cuarto capítulo, el pontífice entra a tallar en un tema que leído desde nuestra realidad nacional, concreta y reciente, permite resaltar en un tono que sabe a consuelo de padre y pastor, entrañable y solidario con nuestro episcopado peruano que, en los Andes y la Amazonía, entre el mundo de algunas minas y otros recursos energéticos del Perú, viene mostrándose unido a sus diócesis sufrientes. Es el tema del ambiente y sus conexiones pastorales económicas, sociales, culturales y políticas del desarrollo de los pueblos, sus derechos y sus deberes en el que el Papa se pronuncia de la manera siguiente: "El acaparamiento por parte de algunos estados, grupos de poder y empresas de recursos energéticos no renovables, es un grave obstáculo para el desarrollo de los países pobres. Éstos no tienen medios económicos ni para acceder a las fuentes energéticas no renovables ya existentes ni para financiar la búsqueda de fuentes nuevas y alternativas. La acumulación de recursos naturales, que en muchos casos se encuentran precisamente en países pobres, causa explotación y conflictos frecuentes entre las naciones y en su interior. Dichos conflictos se producen con frecuencia precisamente en el territorio de esos países, con graves consecuencias de muertes, destrucción y mayor degradación aún. La comunidad internacional tiene el deber imprescindible de encontrar los modos institucionales para ordenar el aprovechamiento de los recursos no renovables, con la participación también de los países pobres, y planificar así conjuntamente el futuro." (CV.49)

Benedicto XVI, introduce su encíclica afirmando en sus primeras tres líneas, dos ideas claves que ampliará y desarrollará en el conjunto de los seis extensos capítulos de su encíclica: de un lado sostiene que, "la caridad en la verdad es la principal fuerza impulsora del auténtico desarrollo de cada persona y de toda la humanidad." (CV.1). Para evitar cualquier 
ambigüedad, sentencia que en definitiva "el amor es una fuerza extraordinaria, que mueve a las personas a comprometerse con valentía y generosidad en el campo de la justicia y de la paz." De otro, que "la caridad es la vía maestra de la doctrina social de la Iglesia, porque no es solo el principio de las micro-relaciones, como en las amistades, la familia, el pequeño grupo, sino también de las macro relaciones, como las relaciones sociales, económicas y políticas."(CV.4) Se trata, entonces, de una preocupación que es sostenida a lo largo de toda la encíclica y que por eso, antes de unirse en coro con Pablo VI, para expresar la ternura filial de la Iglesia que peregrina en la historia invoca a la madre de Jesús como Speculum iustitiae y Regina pacis, es decir, como María, espejo de justicia y Reina de la paz, concluyendo el texto completo con estas expresiones: "Ante el ingente trabajo que queda por hacer, la fe en la presencia de Dios nos sostiene, junto con los que se unen en su nombre y trabajan por la justicia."(CV.78). Cito uno de los fragmentos más representativos por el cual Benedicto XVI, intenta mostrar su cordialidad pastoral para contextos como los de nuestro continente, y concretamente de nuestros Andes y Amazonía: "El acaparamiento de los recursos, especialmente del agua, puede provocar graves conflictos entre las poblaciones afectadas. Un acuerdo pacífico sobre el uso de los recursos puede salvaguardar la naturaleza y, al mismo tiempo, el bienestar de las sociedades interesadas. La Iglesia tiene una responsabilidad respecto a la creación y la debe hacer valer en público. Y, al hacerlo, no sólo debe defender la tierra, el agua y el aire como dones de la creación que pertenecen a todos. Debe proteger sobre todo al hombre contra la destrucción de sí mismo." (CV.51)

Podemos entender de una parte, que esta explícita posición ambientalista del Papa, es la manera profética de expresar de qué caridad y salvación es la que le corresponde testimoniar a la Iglesia. Pero, de otra parte, sobre todo, podemos comprobar que es así, a tiempo y a destiempo, como el Pontífice, al recordarle a la Iglesia su vocación profética y vocación discípulo misionera, le está pidiendo a todas las diócesis de la iglesia universal, que actualicen aquí y ahora su rostro y gesto pastoral. Es por el tratamiento valiente de esta problemática, que a su vez representa el respaldo rotundo de quienes por ejemplo en nuestro país obispos y religiosas, sacerdotes, laicas y laicos- vienen desarrollando esta importante, aunque polémica labor. En definitiva, más allá del alcance e impacto favorable de sus afirmaciones y retos, en esta carta Benedicto XVI, logra transmitir su preocupación y compartir su compromiso por el cuidado de la creación, al grado de no necesitar mencionar la palabra 'pastor' porque él sabe que sólo al auténtico pastor puede moverle y desafiarle hondamente, tanto el deshumanizado funcionamiento de la actual sociedad planetaria, como el hasta ahora tan poco advertido "cristianismo de caridad sin verdad" principalmente.

No basta evangelizar o decir que se hace pastoral, si aquella caridad carece de mordiente social y pública; si la caridad que la impulsa rehúsa la autocrítica y se exime de las exigencias de cambio integral; y del deber evangélico de incidir en la dimensión estructural e institucional con que funciona la sociedad. Y porque, parafraseando la fuente que inspira el perfil del auténtico pastor, aquella caridad que no conjugue con la verdad, puede asociársela más a los motivos del mercenario y del salteador... a los proyectos del ladrón que no viene más que a robar, matary destruir." (Jn 10,1 10). Transcribo: "Un cristianismo de caridad sin verdad se puede confundir fácilmente con una reserva de buenos sentimientos, 
provechosos para la convivencia social, pero marginales -es decir, sin incidencia ni impacto transformador alguno-. De este modo, en el mundo no habría un verdadero y propio lugar para Dios. Sin la verdad, la caridad es relegada a un ámbito de relaciones reducido y privado. Queda excluida de los proyectos y procesos para construir un desarrollo humano de alcance universal, en el diálogo entre saberes y operatividad." (CV.4)

Se trata, desde luego, de un reclamo y de una alerta que sólo podía venir del Papa, el discípulo misionero mayor, que ha puesto la teología al servicio de la vida de los fieles y de sus hermanos discípulos misioneros en general, antes que a otros intereses. Y he aquí, en mi opinión, una de las contribuciones pastorales más oportunas y fecundas que hace el Papa Benedicto XVI, compartiéndonos con esta carta su preocupación de Pastor: hacer recordar al mundo que la misión que Jesucristo le ha encomendado a la Iglesia entera es, -parafraseando al Evangelio-, la de hallarse como luz en el mundo y sal en la tierra. La que, con apremio y cuidado cual vino nuevo en vasijas nuevas, cual levadura en la masa, con la expresa responsabilidad de llevar a cabo las siete consignas veterotestamentarias del verdadero pastor, señaladas por el profeta Ezequiel: "preocuparse por el rebaño disperso, fortalecer a las ovejas débiles, atender a las enfermas, vendar a las heridas, reunir al rebaño, a la oveja apartada, buscar a la perdida" (Ezequiel 34,4-5).

Este desasosiego por el rebaño olvidado, alejado y perdido, resulta salvaguardado y reivindicado por el Papa, cada vez que al hablar de caridad, lo hace para recuperar su "misión de verdad que cumplir en todo tiempo y circunstancia a favor de una sociedad a medida del hombre, de su dignidad y de su vocación" (CV.9) Es decir, clamando por la presencia pública de la Iglesia en esta sociedad $(3,11,56)$, en una posición opuesta, tanto a cierta pretensión a "mezclarse en la política de los Estados" , como a la de reducir su misión-visión a la de la exclusiva beneficencia y ausencia pública, o a la simple función decorativa e incluso a la mera homeóstasis o función de agente estabilizadora reguladora de la sociedad. Expresa: "toda la Iglesia, en todo su ser y obrar, cuando anuncia, celebra y actúa en la caridad, tiende a promover el desarrollo integral del hombre. Tiene un papel público que no se agota en sus actividades de asistencia o educación, sino que manifiesta toda su propia capacidad de servicio a la promoción del hombre y la fraternidad universal cuando puede contar con un régimen de libertad. Dicha libertad se ve impedida en muchos casos por prohibiciones y persecuciones, o también limitada cuando se reduce la presencia pública de la Iglesia solamente a sus actividades caritativas". (CV.11)

Por eso, cuando el Papa toque el tema del desarrollo humano en nuestro tiempo, en el capítulo segundo de la Caritas in veritate, se mostrará como el pastor que, celoso por la integridad de su rebaño, se alarma y lo pone en alerta ante "... la falta de reglamentación del mundo del trabajo. Estos procesos han llevado a la reducción de la red de seguridad social a cambio de la búsqueda de mayores ventajas competitivas en el mercado global, con grave peligro para los derechos de los trabajadores, para los derechos fundamentales del hombre y para la solidaridad en las tradicionales formas del Estado social. Los sistemas de seguridad social pueden perder la capacidad de cumplirsu tarea, tanto en los países pobres, como en los emergentes, e incluso en los ya desarrollados desde hace tiempo. En este punto, las políticas de balance, con los recortes al gasto social, con frecuencia 
promovidos también por las instituciones financieras internacionales, pueden dejar a los ciudadanos impotentes ante riesgos antiguos y nuevos; dicha impotencia aumenta por la falta de protección eficaz por parte de las asociaciones de los trabajadores."(CV.25)

Haciéndose eco de todos sus predecesores, desde la Rerum Novarum de 1891, advierte: "El estar sin trabajo durante mucho tiempo, o la dependencia prolongada de la asistencia pública o privada, mina la libertad y la creatividad de la persona y sus relaciones familiares y sociales, con graves daños en el plano psicológico y espiritual. Quisiera recordar a todos, en especial a los gobernantes que se ocupan en dar un aspecto renovado al orden económico y social del mundo, que el primer capital que se ha de salvaguardar y valorar es el hombre, la persona en su integridad: 'Pues el hombre es el autor, el centro y el fin de toda la vida económico-social'. (CV.25).

En especial, leída su carta desde el subcontinente nuestro, a propósito de sus graves dolencias estructurales, Benedicto XVI se está consagrando entre nosotros, como el Papa profeta que denuncia una caridad de sacristía o parroquia, una caridad encadenada o hipotecada que deja la sociedad como está: ajena, o peor, indiferente al curso dramático de la vida cotidiana digna, de las mayorías de nuestra sociedad. El Papa de la caridad que se desprivatiza y libera con el Evangelio. La que así, se despoja de paternalismos, y de relaciones asimétricas y verticalistas, la que deja de ser ajena e indiferente a la lucha por la justicia y los derechos humanos, al desarrollo de todo el hombre y de todos los hombres. La caridad que ha de nutrir el testimonio público de la iglesia y los cristianos, tiene que estar empapada de preocupaciones aparentemente menudas o domésticas, por no decir femeninas, y que el Papa hace muy suyas cuando echa de menos la "...falta - de- un sistema de instituciones económicas capaces, tanto de asegurar que se tenga acceso al agua y a la comida de manera regular y adecuada desde el punto de vista nutricional. "(CV.27)

Desde esta carta, el Papa apuesta por una caridad purificada y examinada en la verdad. Por una presencia eclesial literal y auténticamente pastoral de caridad en la verdad, que para salvar el desarrollo de la vida y la dignidad de la persona humana de todo el hombre y de todos los hombres, sale de la especie de refugio blindado que da lo convencional protocolary acostumbrado; se despoja de las posturas supuestamente neutrales ${ }^{45}$, para recuperar su posición pública y reanudar desde ella su cometido salvífico: "La «ciudad del hombre» no se promueve sólo con relaciones de derechos y deberes sino, antes y más aún, con relaciones de gratuidad, de misericordia y de comunión. La caridad manifiesta siempre el amor de Dios también en las relaciones humanas, otorgando valor teologal y salvífico a todo compromiso por la justicia en el mundo."

\section{La vocación, en la Caritas in Veritate del Papa Benedicto XVI}

Otra de las claves importantes con que el Papa inspira y articula el contenido

45 Como pastor cuidadoso, que guía por los buenos pastos a su rebaño, el Papa siembra su carta de una serie de advertencias, entre otras, frente a la seducción de la neutralidad. Con relación a la economía, señala que una de sus "mayores tareas es precisamente el uso más eficaz de los recursos, no el abuso, teniendo siempre presente que el concepto de eficiencia no es axiológicamente neutral." (50). Y con relación a los medios de comunicación social "se han introducido de tal manera en la vida del mundo, que parece realmente absurda la postura de quienes defienden su neutralidad y, consiguientemente, reivindican su autonomía con respecto a la moral de las personas. (73) 
central de su Caritas in veritate es la convicción de que el trabajo, el desarrollo, la naturaleza y hasta el mismo ser y actuar de la Iglesia, no son sino, un palpitante asunto de vocación. Es decir, no tienen otra explicación y otra razón de ser que la pura iniciativa, generosa llamada y gratuito obsequio del Señor. Refiriéndose por ejemplo a la técnica, el Papa dice que ella "... responde a la misma vocación del trabajo humano: en la técnica, vista como una obra del propio talento, el hombre se reconoce a sí mismo y realiza su propia humanidad." (CV.69)

Pensando en el desarrollo, afirma que "La verdad, y el amor que ella desvela, no se pueden producir, sólo se pueden acoger. Su última fuente no es, ni puede ser, el hombre, sino Dios, o sea Aquel que es Verdad y Amor. Este principio es muy importante para la sociedad y para el desarrollo, en cuanto que ni la Verdad ni el Amor pueden ser sólo productos humanos; la vocación misma al desarrollo de las personas y de los pueblos no se fundamenta en una simple deliberación humana, sino que está inscrita en un plano que nos precede y que para todos nosotros es un deber que ha de ser acogido libremente." (CV.52) De otro, al referirse a la naturaleza afirma que "también ella, es una «vocación» (CV.48), porque"...es expresión de un proyecto de amor $y$ de verdad. Ella nos precede y nos ha sido dada por Dios como ámbito de vida. Nos habla del Creador (cf. Rm 1,20) y de su amor a la humanidad. Está destinada a encontrar la «plenitud» en Cristo al final de los tiempos (cf. Ef. 1,9-10; Col 1,19-20).

Benedicto XVI, hace estas perspicaces afirmaciones teológicas, retomando la inspiración teológica del Papa Pablo VI y su aún vigente Populorum Progressio, después de haber fundamentado en su primer capítulo, que "el progreso, en su fuente y en su esencia, es una vo- cación. Porque «en los designios de Dios, cada hombre está llamado a promover su propio progreso, ya que la vida de todo hombre es una vocación» (CV. 16). Con el Papa que sucedió al actual Beato Juan XXIII, no sólo reitera que el desarrollo es vocación, porque este nace de una llamada trascendente, y que la vocación es una llamada que por necesitar de una respuesta libre y responsable, resulta salvaguardando la verdadera autonomía de la persona. Es esa misma libertad, la soberanía del pastor dispuesto a dar la vida por su rebaño la que ha preparado a Benedicto XVI para converger en una consideración grave y explosiva que nos involucra y ampara, al unísono y como en un solo cuerpo con todos los Papas que concurrieron desde León XIII hasta Pablo VI y Juan Pablo II: señalar de manera profética que la situaciones de subdesarrollo por las que transitamos como país y como continente, obedecen no a casualidades o fatalidades, sino a causas y responsabilidades, habitualmente atribuidas injustamente a las mismas víctimas de estos procesos. Esta libertad se refiere al desarrollo que tenemos ante nosotros pero, al mismo tiempo, también a las situaciones de subdesarrollo, que no son fruto de la casualidad o de una necesidad histórica, sino que dependen de la responsabilidad humana. Por eso, 'los pueblos hambrientos interpelan hoy, con acento dramático, a los pueblos opulentos'. También esto es vocación, en cuanto llamada de hombres libres a hombres libres para asumir una responsabilidad común.

El Papa señala que la hermandad, expresión del vínculo profundo entre las personas y los pueblos, "nace de una vocación trascendente" (CV.18), que "la fe cristiana se ocupa del desarrollo, no apoyándose en privilegios o posiciones de poder, ni tampoco en los méritos de los cristianos, que ciertamente se han dado 
y también hoy se dan, junto con sus naturales limitaciones, sino sólo en Cristo, al cual debe remitirse toda vocación auténtica al desarrollo humano integral."

Intentando comprender la relación que puede existir entre las tareas del pastor y este énfasis que hace el Papa en decir que el respeto y aprecio por la tierra, la naturaleza, el desarrollo, son un asunto de vocación divina y una cuestión de llamada de Dios, lo que hice fue revisar el texto del evangelio de Juan 10. Ahí, me pareció hallar sugerido el significado de vocación y llamado en la voz, la persona y la misma integridad de Jesús, pastor de pastores y rebaños, que nos identifica y nos llama, que se nos vuelve esa voz conocida que toca hondo y se convierte en umbral y acceso, puerta y puerto al que podemos acceder tras la salvación. "En verdad, -dice Jesús- en verdad les digo: el que no entra por la puerta en el redil de las ovejas, sino que escala por otro lado, ése es un ladrón y un delincuente; pero el que entra por la puerta es pastor de las ovejas. A éste le abre el portero, y las ovejas escuchan su voz; y a sus ovejas las llama una por una y las saca fuera. Cuando ha sacado todas las suyas, va delante de ellas, y las ovejas le siguen, porque conocen su voz. Pero no seguirán a un extraño, sino que huirán de él, porque no conocen la voz de los extraños.» Jesús les dijo esta parábola, pero ellos no comprendieron lo que les hablaba." (Juan 10, 1-6)

En definitiva, hasta aquí, hay que concluir que tanto la riqueza de la experiencia de la caridad, como la fuente y el destino de la vocación para disfrutarla, encuentran en la identidad del pastor abnegado y de su voz de alta fidelidad, el impulso para conseguir un funcionamiento menos deshumanizado y excluyente de la sociedad; la clave con que en Perú, la Iglesia nutre y mantiene invictas y resistentes las comunidades cristianas de la mayoría de nuestras diócesis.

Concluyo, comentando que al fin me pareció comprender el por qué de su deliberado no preferir mencionar en esta carta, ni una sola vez la palabra 'pastor' o 'reino de Dios'. Acaso, porque Benedicto XVI, simplemente se propuso poner su pluma, su voz y su misma vida a disposición de la única Voz y Palabra del único Pastor. Y que, valiéndose de la orden del discípulo misionero convertido en apóstol, que tras su caída en Damasco, escribió insistente ayer, con él se alzan ambos hoy, Pablo el apóstol y Benedicto XVI el actual Papa, para continuar interpelándonos como lo hiciera el primero con las primeras comunidades, advirtiéndonos con gravedad y confianza: "Que su caridad no sea más una farsa". (Rom 12,9).

\section{REFERENCIAS}

Benedicto XVI. (2009). Caritas in Veritate. Lima-Perú, Paulinas.

Benedicto XVI. (2007). Spe Salvi. LimaPerú, Paulinas.

Benedicto XVI. (2006). Deus Caritas est. Lima- Perú, Paulinas

Biblia de Jerusalén. Bilbao. Editorial Desclee De Brouwer.

Pablo VI. (1997). Gaudium Et Spes. Sobre la Iglesia en el mundo de hoy. Lima, Paulinas

Pablo VI. (1967). Populorum Progressio. Lima, Paulinas.

Ratzinger, J. (2007). Jesús de Nazareth. Colombia, Editorial Planeta. 